Article

\title{
Common genetic variants shared among five major psychiatric disorders: a large-scale genome-wide combined analysis
}

\author{
Lu Xia1,2, Kun Xia2,6, Daniel R Weinberger33, Fengyu Zhang1,4,5* \\ ${ }^{1}$ Global Clinical and Translational Research Institute, Bethesda, MD, USA; \\ ${ }^{2}$ Center for Medical Genetics \& Hunan Key Laboratory for Medical Genetics, College of Life Sciences, the Central South \\ University, Changsha, Hunan, China; \\ ${ }^{3}$ Lieber Institute for Brain Development, Department of Psychiatry and Behavioral Sciences, Neurology, Neuroscience, \\ Institute of Genomic Medicine, Johns Hopkins University School of Medicine, Baltimore, MD, USA; \\ 4The Second Xiangya Hospital \& National Clinical Research Center for Mental Health Deriders, Central South University, \\ Changsha, Hunan, China; \\ 5Peking University Huilongguan Clinical Medical School \& Beijing Huilongguan Hospital, Beijing, China; \\ ${ }^{6}$ Chinese Academy of Sciences Center for Excellence in Brain Science and Intelligences Technology (CENSIT), Shanghai, \\ China.
}

Received Feburary 7, 2019; Accepted Febueary 27, 2019

\begin{abstract}
Background: Genetic correlation and pleiotropic effects among psychiatric disorders have been reported. This study aimed to identify specific common genetic variants shared between five adult psychiatric disorders: schizophrenia, bipolar, major depressive disorder, attention deficit-hyperactivity disorder, and autism spectrum disorder.

Methods: A combined p value of about 8 million single nucleotide polymorphisms (SNPs) were calculated in an equivalent sample of 151,672 cases and 284,444 controls of European ancestry from published data based on the latest genome-wide association studies of five major psychiatric disorder using Stouffer's Z-score method. SNPs that achieved genome-wide significance $\left(\mathrm{P}<5 \times 10^{-08}\right)$ were mapped to loci and genomic regions for further investigation; and gene functional annotation and clustering were performed to understand biological process and molecular function of the loci identified. We also examined CNVs and performed expression quantitative trait loci analysis for SNPs by genomic region.

Results: We find that 6,293 SNPs mapped to 336 loci are shared by the three adult psychiatric disorders, 1,108 variants at 73 loci are shared by the childhood disorders, and 713 variants at 47 genes are shared by all five disorders at genome-wide significance $\left(\mathrm{p}<5 \times 10^{-08}\right)$. Of the 2,583 SNPs at the extended major histocompatability complex identified for three adult disorders, none of them were associated with two childhood disorders; and SNPs shared by all five disorders were located in the regions that have been identified as containing copy number variation associated with autism and had largely neurodevelopmental functions.

Conclusion: We show a number of specific SNPs associated with psychiatric disorders of childhood or adult onset, illustrating not only genetic heterogeneity across these disorders but also developmental genes shared by them all. These results provide a manageable list of anchors from which to investigate epigenetic mechanism or gene-gene interaction on the development of neuropsychiatric disorders and for developing a measurement matrix for disease risk that could potentially be used for new taxonomy for precision medicine.
\end{abstract}

\section{KEYWORDS}

Psychiatric disorders; schizophrenia; bipolar disorder; major depressive disorder; attention deficit-hyperactivity disorder; autism spectrum disorder; genome-wide association study; combined analysis.

\section{INTRODUCTION}

The genome-wide association study (GWAS) has emerged as a compelling tool for investigating the genetic architecture and the etiology of complex human diseases over the past decade[1]. Many common genetic variants have been associated with complex human disorders through GWAS since the early studies in type 2 diabetes and inflammatory bowel disease[2, 3]. As the application of the GWAS approach has progressed, more and more gen-

*Correspondence: Fengyu Zhang, zhangfy@gcatresearch.org, or Kun Xia, xiakun@sklmg.edu.cn 
ome-wide genotyped data have been accumulated, whic$\mathrm{h}$ has made it possible to conduct genome-wide meta-analyses of multiple cohorts of GWAS samples[4]. The number of genome-wide association studies that involve a large number of patients and healthy controls are increasing every year. As of 2017 , about $40 \%$ of recent studies are with a sample size of more than 50,000 individuals [5], and some even with more than 200,000 [6,7]. With samples of such size, investigators expect to identify variants with lower frequency and smaller effect size, by overcoming genetic heterogeneity[8] to attain adequate power to detect a genetic association. More importantly, with the development of genotype imputation and large whole-genome sequencing datasets available, it is feasible to assess the whole genome common variants for association with common complex human disorders without whole genome sequencing of all sample individuals.

The lessons from GWAS include that association of genetic variants with common human disorders is complex and involves a matrix of polygenic and pleiotropic effects. It has become clear that common complex human disorders are affected by multiple variants in a polygenic way [9]; whereas an individual variant may be associated with multiple diseases or traits (i.e., pleiotropic). Preliminary evidence of pleiotropic effects has been found in immune-related disorders, various types of cancers, or neuropsychiatric disorders[10]. For example, genetic varints at 3p21,10q24, and SNPs within two L-type voltage-gated calcium channel subunits, $C A C N A 1 C$ and $C A C N B 2$ have been found to be shared by multiple psychiatric disorders [11]. Coincidentally, genome-wide SNPs association analysis has revealed that psychiatric disorders might share a moderate to high degree of genetic correlation [12]. Identification of the pleiotropic effects of specific genetic variants on common complex human disorders is a potentially important step in building the knowledge network and a measurement matrix for disease risk for developing a new taxonomy of human diseases, which will play a fundamental role in achieving the goals of precision medicine $[13,14]$.

\section{METHODS}

Dataset and analysis. Individual SNP data used in this study were obtained from the published data releases by the Psychiatric Genomic Consortium that contained the largest genome-wide association studies of schizophrenia (SCZ), bipolar disorder (BD) [15], and major depressive disorder (MDD) [7], attention deficit hyperactivity disorder (ADHD) [16], and autism spectrum disorder (ASD) [17]. Detailed information on the sample individuals, original data process and analysis of individual sample cohort has been described previous in the individual studies. Of note, the ADHD sample mainly include a population-based cohort of ADHD cases and controls fro$m$ Denmark collected by the Lundbeck Foundation Initiative for Integrative Psychiatric Research (iPSYCH), and 11 cohorts aggregated by the Psychiatric Genomics Consortium (PGC); the majority of the ASD sample was also from iPSYCH ASD and five family-based samples of Euro- pean ancestry, which contributed cases and pseudocontrols.

The analysis was performed by calculating the combined $p$ values of individual SNPs across the genome in multiple disorders. The Stouffer's Z-score method (1949), an extension of classical Fisher's combined method (1925) that has been employed in previous GWAS [18], was used to have a two-sided test. The hypothesis would be "multiple psychiatric disorders shared some common genetic variants," and under a null hypothesis, a statistic can be calculated by a combined Z-score, which can be converted into p-value based on the inverse of the standard normal cumulative distribution. The Z-score is defined as $\mathrm{Z}=$ $\frac{\sum_{i=1}^{k} Z_{i}}{\sqrt{k}}$; where $\mathrm{k}$ is the number of individual disorders, and the $Z_{i}$ is the inverse of the standard normal cumulative distribution based on the $\mathrm{p}$ value for an individual SNP in the $i$ th disorder.

The analysis was limited to SNPs showing a consistent direction of association at nominal significance $(\mathrm{P}<0.05)$ across disorders, to keep internal consistency and reduce heterogeneity, i.e., ruling out SNPs, which is highly significant in one disorder, but not the other or shows opposite allelic directionality across disorders. The combined analysis was performed first for schizophrenia, bipolar and major depressive disorder, i.e., the three adult psychiatric disorders that have been demonstrated a moderate to high genetic correction, then for ADHD and ASD, the two childhood neurodevelopmental disorders, and finally for five disorders combined. A genome-wide significance threshold $\left(\mathrm{P}<5 \times 10^{-08}\right)$ was employed.

SNP functional annotation and eQTL analysis. Individual SNPs were mapped to a locus based on the dbSNP and the HapMap data[19], and then the unmapped SNPs by the database were manually verified with the UCSC genome browser (GRCh37/hg19). Functional annotation of associated loci was performed based on the DAVID Bioinformatics Resource 6.8 using Gene Ontology (GO) term [20]. The eQTL analysis was performed on the GTEx dataset developped by the Genotype-Tissue Expression (GTEx) Project; the gene expressions in human tissues were based on the Human Protein Atlas (HPA) RNA-seq (https://www.proteinatlas.org/).

\section{RESULTS}

Table 1 presents the number of sample individuals and SNPs included in this study by the individual disorder. Briefly, the schizophrenia and bipolar analysis consisted of 53,555 cases (20,129 BD, 33,426 SCZ) and 54,065 controls with 8,379,106 SNPs; the MDD study comprised 59,851 cases and 113,154 controls with 13,554,492 SNPs; the ADHD study included 20,183 cases and 35,191 controls with 8,047,420 SNPs; and the ASD study included 9,112,386 SNPs in 18,381ASD cases and 22,664 controls. The combined $p$ values were calculated to identify genetic variants shared by multiple disorders (Methods). While the original data in the MDD study included a fair 
number of rare variants, we only focused on the common variants (minor allele frequency, $\mathrm{MAF}>1 \%$ ).

Table 1. Samples and number of SNPs by individual disorder

\begin{tabular}{llrrr}
\hline Disorder & Sample & No. variants & Cases & Control \\
\hline Schizophrenia (SCZ) & PGC & $8,379,106$ & 33,426 & 54,065 \\
Bipolar disorder (BD) & PGC & $8,958,989$ & 20,129 & 54,065 \\
Major depressive disorder(MDD) & PGC & $13,554,492$ & 59,851 & 113,154 \\
Attention deficit hyperactivity disorder(ADHD) & Combined & $8,047,420$ & 20,183 & 35,191 \\
& iPSYCH* & & 14,584 & 22,492 \\
& PGC-ADHD & 5,499 & 12,599 \\
Autism spectrum disorder (ASD) & Combined & $9,112,386$ & 18,381 & 27,969 \\
& iPSYCH & & 13,076 & 22,664 \\
Common SNPs in combined five disorders & PGC -ASD & 5,305 & 5,305 \\
\hline
\end{tabular}

*Cases and controls from Denmark collected by the Lundbeck Foundation Initiative for Integrative Psychiatric Research (iPSYCH);

PGC-ASD, Psychiatric Genomic Consortium--Autism spectrum disorders contained five family-based trio samples of European ancestry; PGC-ADHD, Psychiatric Genomic Consortium--Attention deficit and hyperactivity disorder comprised 11 European, North American and Chinese cohorts aggregated by the Psychiatric Genomics Consortium.

Common variants associated with schizophrenia, bipolar and major depressive disorder

We identified 6,293 SNPs shared by three adult-onset disorders at genome-wide significance $\left(\mathrm{P}<5 \times 10^{-08}\right)$. These included 2,583 SNPs in the extended major histocompatability complex (xMHC), which spans a 7.5-Mb region defined by the SLC17A2 gene at the telomeric end to the $D A X X$ gene at the centromeric end of chromosome 6 (Chr6:25,875,084-33,797,216, hg19) and mapped to 421 loci (excluding tRNA genes)according to the gene map of

A. SCZ+BD+MDD

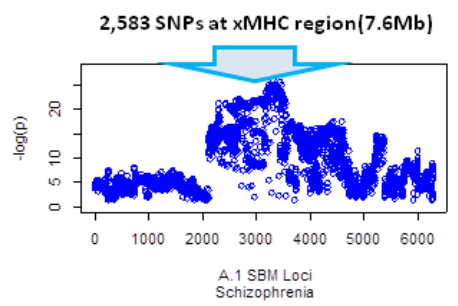

2,583 SNPs at $\mathrm{XMHC}$ region (7.6Mb)

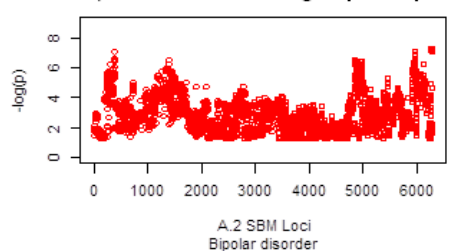

A.2 SBM Loci
Bipolar disorder

2,583 SNPs at $x$ MHC region (7.6Mb)

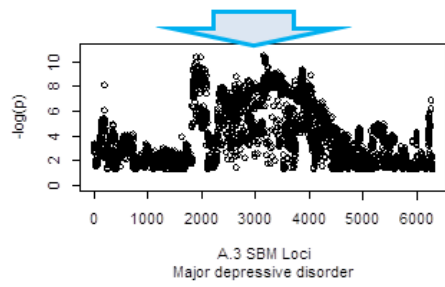

xMHC [21, 22]; and 3,710 SNPs were located in the nonxMHC (Figure 1A). We noted that these SNPs were in strong linkage disequilibrium (LD) and clustered by genomic region. Of the total non-xMHC SNPs, 3,654 SNPs (98.5\%) were mapped to 68 genomic regions, defined by which flanking SNPs of any two regions were at least 1$\mathrm{Mb}$ apart. Based on the SNP functional prediction[19], 263 SNPs ( $8 \%$ ) were at TFBS, and 109 SNPs (3.3\%) were conserved in vertebrates (Table S2).
B. ADHD + ASD
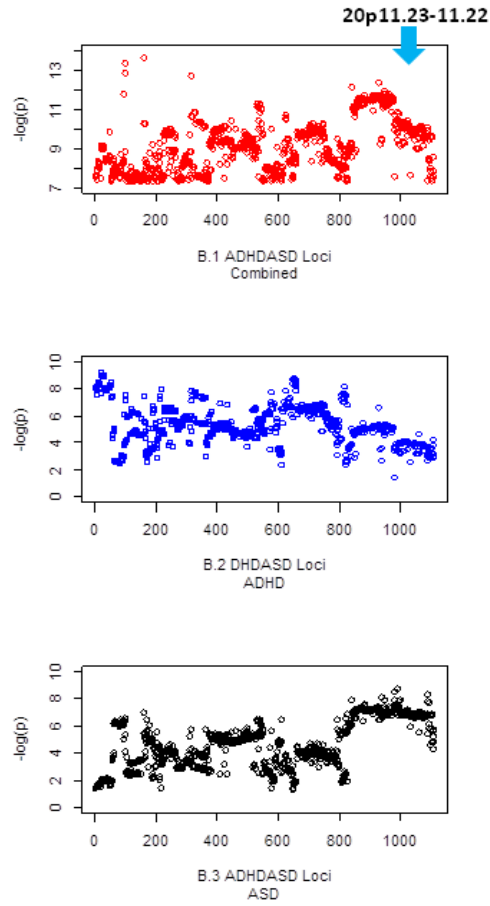

Figure 1. Scatter plots of $p$ values (-log) for SNPs shared by psychiatric disorders. (x-Axis is SNPs in chromosomal order; A.1-A.3, 6,293 SNPs shared by three adult-onset disorders for association with an individual disorder; B.1-B.3, 1,108 SNPs shared by ADHDASD and for associ-ation with ADHD and ASD). 
Among the 68 regions at non-xMHC loci, 51 regions did not have an SNP associated with a particular disorder at genome-wide significance; whereas other 17 regions cotained at least one SNPs in each region associated with an individual disorder at genome-wide significance. In the 51 regions that might be novel or specifically shared by the three adult-onset disorders (Table 2), 15 regions contained only a single SNPs; these included chr1: 163646390 at NUF2, rs7581403 at MYT1L, chr2:185676179 at ZNF804A, chr2:193934836, rs13078307 at CNTN4, rs7613933 at WDR49,chr5:152167615, rs10239423 at SP4, rs7905569 at IFITM8P, rs4837673 at DBC1//MIR147, rs1828385 at LOC100120660//LOC751600, chr18:
26337461, rs72903982 at KC6, chr20:43682549 at STK4, chr20:48108321 at RYR2; and the other 36 regions contained about 80 genes, including DAMTSL3, $A L P K 3, A N K$ S1B, BRAF, COX8A, DCC, ENOX1, FGFR1, GABRA1, GABRG2, GCKR, GRIN2A, KCNB1, KCTD13, NFIX, PCLO, TRIM8, and BORCS7. Meanwhile, SNPs in the other 17 genomic regions, each containing at least one SNP associated with an individual disorder at genome-wide significance, were mapped to 85 genes; and some of which CACNB2, DRD2, CACNA1C, CACNAI1, AS3MT, TSNARE1, KLC1, and SOCRS3 have been associated with schizophrenia, and EP300, VRK2, BAG5, APOPT1 have been associated with MDD at genome-wide significance (Table 2) .

Table 2. Genomic regions and loci mapped by the 6,293 SNPs $(3,710$ at non-xMHC) shared by three adult-onset psychiatric disorders at genome-wide significance.

\begin{tabular}{|c|c|c|c|c|c|c|c|c|}
\hline Locus & & $\mathrm{Chr}$ & SNP & BP1 & $\mathrm{BP} 2$ & ID & \#SNP & Mapped gene loci \\
\hline 1 & & 1 & $\mathrm{rs} 200448$ & 6701978 & 6765079 & 1 & 21 & DNAJC11, CAMTA1, \\
\hline 2 & & 1 & rs4949240 & 30438197 & 30509225 & 22 & 38 & PTPRU/|MATN1 \\
\hline 3 & ** & 1 & rs2841192 & 73307627 & 73856328 & 60 & 132 & KRT8P21, LRRC44 \\
\hline 4 & & 1 & rs6661796 & 79186203 & 79261383 & 192 & 43 & IFI44||ADGR4L, ELTD1 \\
\hline 5 & & 1 & chr1_16364 & 163646390 & & 251 & 1 & NUF2 \\
\hline 6 & ** & 1 & rs 11587347 & 239198959 & 244025999 & 252 & 5 & [RGS7, KMO, OPN3, WDR64, EXO1, PLD5], ZNF238 \\
\hline 7 & & 2 & rs7581403 & 2314997 & 2314997 & 257 & 1 & MYT1L \\
\hline 8 & & 2 & chr2_22499 & 22499207 & 28290347 & 258 & 458 & BRE, C2orf16, GCKR, GPN1, RBKS, SUPT7L, KLHL29, MRPL32, MRPL33, ZNF512 \\
\hline 9 & ** & 2 & rs13011472 & 57961602 & 58308458 & 717 & 10 & VRK2 2 \\
\hline 10 & & 2 & rs56088823 & 96793024 & 96905568 & 727 & 13 & ASTL, DUSP2, STARD7 \\
\hline 11 & & 2 & chr2_18567 & 185676179 & & 740 & 1 & ZNF804A \\
\hline 12 & & 2 & chr2_19393 & 193934836 & & 741 & 1 & NA \\
\hline 13 & & 2 & chr2_19822 & 198226403 & 198940251 & 742 & 206 & BOLL, MARS2, PLCL1, RFTN2 \\
\hline 14 & & 2 & rs13020196 & 201071942 & 201244261 & 948 & 56 & ERGICZ, SPATS2L \\
\hline 15 & & 2 & rs2551656 & 208371637 & 208531683 & 1005 & 84 & CREB1, FAM119A \\
\hline 16 & & 3 & rs13078307 & 2565355 & 2565355 & 1090 & 1 & CNTN4 \\
\hline 1 & ** & 3 & rs6802320 & 52241835 & 53475074 & 1091 & 523 & $\begin{array}{l}\text { ALAS1, DCP1A, DNAH1, GLTBD1, GLYCTK, GNL3, ITIH1, ITIH3, ITIH4, MUSTN1, SFMBT1, } \\
\text { TWF2, RFT1, MIR135A1, NEK4, NT5DC2, PBRM1,PHF7, WDR82, SPCS1, STAB1, TLR9, TMEM110 }\end{array}$ \\
\hline 18 & & 3 & rs2176028 & 80655077 & 80693369 & 1613 & 6 & [ROBO1, GBE1] \\
\hline 19 & & 3 & rs4146338 & 117493964 & 117772036 & 1619 & 84 & LOC728873/IIGSF11 \\
\hline 20 & & 3 & rs7613933 & 167304189 & 167304189 & 1703 & 1 & WDR49 \\
\hline 21 & & 4 & rs56089943 & 48342682 & 48496368 & 1704 & 41 & SLC1OA4,TEC//SLAIN2, ZAR1 \\
\hline 22 & & 4 & rs1599125 & 118644885 & 118753686 & 1745 & 82 & NT5C3P1//NDST3 \\
\hline 2 & ** & 5 & rs7716818 & 103684787 & 104089064 & 1827 & 271 & NUDT12||RAB9P1 \\
\hline 24 & & 5 & chr5_15216 & 152167615 & 152167615 & 2098 & 1 & AK123826 \\
\hline 25 & & 5 & rs2290732 & 161324898 & 161352075 & 2099 & 14 & GABRA1, GABRG2 \\
\hline 26 & ** & 6 & rs2744301 & 25323143 & 25875567 & 2113 & 59 & CARMIL1, SCGN, HIST1H2AA, SLC17A1, SLC17A3, HIST1H2APS2, SLC17A4 \\
\hline 27 & & 6 & rs 36014129 & 25884519 & 33797216 & 2172 & 2583 & Extended MHC region \\
\hline 28 & & 6 & rs28360639 & 50783501 & 50936376 & 4755 & 71 & FTHP1, RPS17L4,TFAP2B,TFAP2D \\
\hline 29 & & 6 & rs9360557 & 73132745 & 73155701 & 4826 & 14 & RIMS1, [KCNQ5] \\
\hline 30 & & 6 & rs12190758 & 93148341 & 93176175 & 4841 & 4 & [EPHA7] \\
\hline 31 & & 7 & rs10239423 & 21503450 & 21503450 & 4845 & 1 & SP4 \\
\hline 32 & & 7 & rs4291157 & 24624449 & 24844736 & 4846 & 67 & BLVRA, MRPS24, DFNA5, MPP6, OSBPL3 \\
\hline 33 & & 7 & rs2371213 & 82397302 & 82482281 & 4914 & 35 & PCLO \\
\hline 34 & & 7 & rs10265001 & 140665521 & 140777030 & 4950 & 43 & BRAF, MRPS33 \\
\hline 35 & & 8 & rs6983972 & 9699144 & 9728595 & 4994 & 5 & MIR597|| MIR124-1 \\
\hline 36 & & 8 & chr8_33747 & 33747954 & 34021138 & 4999 & 5 & DUSP26 \\
\hline 37 & & 8 & rs57709857 & 38290424 & 38291844 & 5004 & 2 & FGFR1 \\
\hline 38 & & 8 & rs790569 & 64621828 & 64621828 & 5006 & 1 & IFITMBP \\
\hline 39 & ** & 8 & rs10098073 & 143309504 & 143352779 & 5007 & 46 & TSNARE1 \\
\hline 40 & & 9 & rs9695226 & 22759396 & 22785141 & 5053 & 8 & FLJ35282 \\
\hline 41 & & 9 & rs4837673 & 122546769 & 122546769 & 5061 & 1 & DBC1// MIR147 \\
\hline 42 & ** & 10 & rs12777923 & 18661160 & 18775255 & 5062 & 81 & CACNB2 \\
\hline 43 & ** & 10 & rs7893954 & 104318966 & 105059896 & 5143 & 233 & $\begin{array}{l}\text { ARL3, ASSMT, CNNM2, WBP1L, CYP17A1, NT5C2, INA, PCGF6, CXCL12, } \\
\text { SFXN2, SUFU, TRIM8, BORCS7 }\end{array}$ \\
\hline 44 & & 10 & rs12761679 & 106512727 & 106747354 & 5376 & 19 & SORCS3 \\
\hline 45 & & 11 & rs7946546 & 63595648 & 63790521 & 5395 & 83 & ATP5LP1,MARK2,MACROD1, RCOR2, NAA40, COX8A, OTUB1 \\
\hline 46 & ** & 11 & rs17601612 & 113317745 & 113412443 & 5478 & 25 & DRDL, TMPRSSS \\
\hline 47 & & 11 & rs402320 & 131374917 & 131399805 & 5504 & 75 & RREB1 \\
\hline 48 & ** & 12 & rs740416 & 2499892 & 2514270 & 5579 & 8 & CACNA1C \\
\hline 49 & & 12 & rs1319892 & 99436519 & 99690240 & 5587 & 127 & ANKS1B \\
\hline 50 & ** & 12 & chr12_1236 & 123639761 & & 5714 & 1 & SSPN \\
\hline 51 & & 13 & rs9562504 & 44244876 & 44351241 & 5715 & 11 & ENOX1 \\
\hline 52 & & 14 & rs 28645341 & 30174078 & 30182920 & 5726 & 3 & PRKD1 \\
\hline 53 & & 14 & rs36341 & 72390689 & 72454646 & 5729 & 14 & RGS6, SIPA1L1 \\
\hline 54 & ** & 14 & rs11160502 & 99667179 & 99731731 & 5743 & 4 & $B C L 11 B$ \\
\hline 55 & ** & 14 & chr14_1039 & 103916280 & 104319989 & 5747 & 247 & BAG5, APOPT1, KLC1, TRMT61A, CKB, XRCC3, PPP1R13B, ZFYVE21 \\
\hline 56 & & 15 & rs1828385 & 36355868 & & & 1 & LOC100130660||LOC751603 \\
\hline 57 & & 15 & rs2562774 & 84380096 & 85393240 & 5924 & 65 & ADAMTSL3, ALPK3, ZSCAN2, SEC11A, WDR73, ZNF592, \\
\hline 58 & ** & 15 & rs7168951 & 91406146 & 91437388 & 5989 & 24 & $\begin{array}{l}\text { BLM //FURIN,FES,FURIN } \\
\text {, }\end{array}$ \\
\hline 59 & & 16 & rs11648559 & 9874699 & 9960879 & 6013 & 156 & GRIN2A \\
\hline 60 & & 16 & rs12919683 & 29943367 & 30018500 & 6170 & 47 & INOBOE, DOC2A, КCTD13, ТАOK2 \\
\hline 61 & & 18 & chr18_2633 & 26337461 & & 6217 & 1 & NA \\
\hline 62 & & 18 & rs 72903982 & 39152991 & & 6218 & 1 & KC6 \\
\hline 63 & & 18 & rs7505145 & 50711776 & 50870391 & 6219 & 30 & $D C C$ \\
\hline 64 & & 18 & rs56096694 & 52722378 & 53101598 & 6249 & 8 & MAP1LC3P, TCF4 \\
\hline 65 & & 19 & rs17706798 & 13116172 & 13122612 & 6257 & 4 & NFIX \\
\hline 66 & & 20 & chr20_4368 & 43682549 & & 6261 & 1 & STK4 \\
\hline 67 & & 20 & chr20_4582 & 45829133 & & 6262 & 1 & $\begin{array}{l}\text { SYR } 2 \\
\text { RYR }\end{array}$ \\
\hline 68 & & 20 & chr20_4810 & 48108321 & 48110279 & 6263 & 4 & KCNB1//PTGIS \\
\hline 69 & ** & 22 & rs71799331 & 39974015 & 41617897 & 6267 & 27 & CACNA1I, EP300, L3MBTL2, MCHR1/|SLC25A17, MKL1 \\
\hline
\end{tabular}

**, loci where SNPs were associated with one of three disorders; \#SNP, number of SNPs shared by three adult-onset psychiatric disorder at genome-wide significance in each genomic region; [ ], genes nearby; NA, no gene available within about 1- Mb genomic region; SNP is the first flanking SNPs of individual region; BP1 and BP2 are position of two flanking SNPs of individual gene region.

Functional annotation of the genes in the non-xMHC indicated a few top biological processes, including urate met- abolic process, protein phosphorylation, and sodium-dependent phosphate transport. These loci are located at a- 
xon, postsynaptic membrane and postsynaptic density, mitochondrion, cell junction, voltage-gated calcium channel complex, and mitochondrial inner membrane; and they have molecular functions of sodium phosphate symporter activity, sodium-dependent phosphate transmembrane transporter activity, protein binding, p53 binding, ephrin receptor binding, and voltage-gated calcium channel activity involved in AV node cell action potential. However, few of these clustering survived the correction for multiple testing (Table S3).

\section{ADHD and ASD shared loci}

Our combined analysis of 8,047,420 SNPs in 38,266 cases with ADHD or ASD and 63,160 controls identified 1,108 SNPs shared by the two childhood psychiatric disorders at genome-wide significance (Figure 1B). These SNPs were located at 47 genomic regions, of which flanking SNPs of any two adjacent regions were at least $1-\mathrm{Mb}$ apart. Majority of these 1,108 SNPs (88\%) were located at loci 1p34.2, 1p21.3, 4q24, 5q14.3, 5q21.2, 6q13, 7q31.1, 10q25.1, 13q31.1, 16q22.2, 17q21.31, 20p11.23-11.22
(Table 3). All these loci have been reported to contain copy number variants (CNVs) associated with ASD in multiple reports or different study populations, according to the Simons Foundation Autism Research Initiative (SFARI) CNV database (Table S4), The associated genes with these CNVs included HIVP3, RIMS3, DYPD, PTBP2, TET2, $M E F 2 C, R I M S 1$, and SLC25A39. In addition, of the total 47 genomic regions identified in this analysis, five regions at 1p21.3, 5q14.3, 16q22.2, 10q25.1,20p11.23-11.22 contained at least one SNP associated with either ADHD or ASD at genome-wide significance. The remain-ing 42 regions that did not have an SNP associated with an individual disorder at GWAS significance may harbor novel loci for ADHD and ASD. The detailed estimates of SNPs with a minimum $p$-value at each genomic region are in the supplementary data (Table S5). However, we did not find any SNPs shared by ADHD and ASD in the xMHC where a large number of SNPs in strong LD were found shared by the three adult-onset disorders.

Table 3. Genomic regions and loci mapped by the 1,108 SNPs common to ADHD and ASD at genome-wide significance.

\begin{tabular}{|c|c|c|c|c|c|c|c|c|c|c|}
\hline Region & ID & $\mathrm{Chr}$ & SNP1 & BP1 & SNP2 & BP2 & $\# \mathrm{~S}$ & Mapped genes & CNVs & \\
\hline 1 & 1 & 1 & rs35947542 & $43,983,679$ & rs3952787 & $44,323,244$ & 54 & JMJD2A,PTPRF,ST3GAL3 & $1 \mathrm{p} 34.2$ & \\
\hline 2 & 55 & 1 & rs61783205 & $46,317,219$ & rs77881576 & $46,587,530$ & 7 & MAST2 & $1 \mathrm{p} 34.1$ & \\
\hline 3 & 62 & 1 & rs222901 & $96,508,040$ & rs11307310 & $96,998,097$ & 102 & RP11-147C23.1,RP5-898J17.1, РTBP2 & $1 \mathrm{p} 21.3$ & * \\
\hline 4 & 164 & 1 & rs35518820 & $99,035,830$ & rs6662897 & $99,095,611$ & 6 & LOC729987|/SNX7 & & \\
\hline 5 & 170 & 2 & rs76504400 & $1,722,904$ & rs76504400 & $1,722,904$ & 1 & PXDN & & \\
\hline 6 & 171 & 2 & rs77966298 & $10,984,514$ & rs77966298 & $10,984,514$ & 1 & PDIA6 & $2 \mathrm{p} 25.1$ & \\
\hline 7 & 172 & 2 & rs6711582 & $159,327,935$ & rs1548635 & $159,529,888$ & 14 & PKP4 & $2 q 24.1$ & \\
\hline 8 & 186 & 2 & rs75263467 & $174,601,275$ & rs75263467 & $174,601,275$ & 1 & $S P 3$ & & \\
\hline 9 & 187 & 3 & rs74877867 & $20,493,116$ & rs14607701 & $20,621,759$ & 5 & MITF & & \\
\hline 10 & 192 & 3 & rs56842404 & $70,253,808$ & rs62254854 & $70,266,538$ & 2 & RP11-231I13.2,LOC100128160/|FOXP1 & & \\
\hline 11 & 194 & 3 & rs11488172 & $82,320,385$ & rs11488172 & $82,320,385$ & 1 & GBE1 & & \\
\hline 12 & 195 & 3 & rs11710737 & $107,464,170$ & rs7634587 & $107,516,847$ & 4 & $B B X$ & & \\
\hline 13 & 199 & 3 & rs9855048 & $128,163,890$ & rs9855048 & $128,163,890$ & 1 & EEFSEC//DNAJB8 & & \\
\hline 14 & 200 & 3 & rs20033201 & $158,001,670$ & rs11707386 & $158,152,073$ & 3 & LOC730057||MAGI1,RSRC1 & & \\
\hline 15 & 203 & 4 & rs71297516 & $2,768,387$ & rs6422311 & $2,781,240$ & 8 & TNIP2/|SH3BP2 & & \\
\hline 16 & 211 & 4 & rs14510234 & $31,147,972$ & rs20072120 & $31,151,465$ & 4 & RP11-617I14.1 & & \\
\hline 17 & 215 & 4 & rs228619 & $103,569,283$ & rs223504 & $103,635,183$ & 51 & MANBA & $4 q 24$ & \\
\hline 18 & 266 & 5 & rs71613075 & $87,514,778$ & rs2009730 & $87,933,568$ & 95 & CTC-498M16.4,LINC00461,TMEM161B-AS1 & $5 q 14.3$ & * \\
\hline 19 & 361 & 5 & rs2635182 & $92,255,166$ & rs34523 & $92,303,352$ & 6 & CTC-458G6.2 & & \\
\hline 20 & 367 & 5 & rs7716818 & $103,684,787$ & rs323509 & $104,082,179$ & 184 & NUDT12//RAB9P1 & $5 q 21.2$ & \\
\hline 21 & 551 & 5 & rs6862136 & $144,495,743$ & rs6884441 & $144,512,659$ & 4 & LOC100132712//ASSP10 & & \\
\hline 22 & 555 & 6 & rs9342783 & $70,852,493$ & rs3818327 & $70,861,135$ & 16 & COL19A1 & $6 q 13$ & \\
\hline 23 & 572 & 7 & rs4947694 & $52,181,844$ & rs4947694 & $52,181,844$ & 1 & COBL & & \\
\hline 24 & 573 & 7 & rs34080086 & $105,017,329$ & chr7:10506 & $105,064,665$ & 2 & $S R P K 2$ & & \\
\hline 25 & 575 & 7 & rs34291892 & $114,058,731$ & rs7799269 & $114,290,491$ & 16 & FOXP2 & $7 \mathrm{q} 31.1$ & \\
\hline 26 & 591 & 8 & rs34458570 & 745,496 & rs1532744 & 786,916 & 6 & ERICH1-AS1 & & \\
\hline 27 & 597 & 8 & rs4739249 & $21,323,694$ & rs4739249 & $21,323,694$ & 1 & LOC100129163//GFRA2, & & \\
\hline 28 & 598 & 8 & rs11445716 & $144,749,175$ & rs11445716 & $144,749,175$ & 1 & ZNF251 & & \\
\hline 29 & 599 & 9 & rs13440322 & $31,026,272$ & rs28495892 & $31,064,791$ & 9 & LOC100130670/|LOC138412 & $9 \mathrm{p} 21.1$ & \\
\hline 30 & 608 & 10 & rs45595836 & $16,691,399$ & rs45595836 & $16,691,399$ & 1 & RSU1 & & \\
\hline 31 & 609 & 10 & rs12769316 & $104,152,751$ & rs12772374 & $104,156,911$ & 2 & GBF1//NFKB2, NFKB2 & & \\
\hline 32 & 611 & 10 & rs61867293 & $106,563,924$ & rs11192280 & $106,776,925$ & 34 & SORCS3 & $10 \mathrm{q} 25.1$ & * \\
\hline 33 & 645 & 11 & rs5793730 & $95,309,155$ & rs5793730 & $95,309,155$ & 1 & RP11-338H14.1//FAM76B & & \\
\hline 34 & 646 & 12 & rs704067 & $89,726,027$ & rs60474271 & $89,776,845$ & 13 & DUSP6,POC1B,RP11-13A1.3 & & \\
\hline 35 & 659 & 13 & rs 9544757 & $78,821,529$ & rs9530779 & $78,969,536$ & 130 & EDNRB//POU4F1 & $13 q 13.1$ & \\
\hline 36 & 789 & 14 & rs14080258 & $29,419,892$ & rs17638843 & $29,524,041$ & 2 & LOC100128215//PRKD1 & & \\
\hline 37 & 791 & 14 & rs36063234 & $33,409,812$ & rs4981170 & $33,412,996$ & 2 & NPAS3 & & \\
\hline 38 & 793 & 14 & rs11263529 & $94,838,142$ & rs28929474 & $94,844,947$ & 2 & SERPINA1 & & \\
\hline 39 & 795 & 15 & rs11857683 & $87,769,703$ & rs8042805 & $87,779,902$ & 3 & LOC100132083//TMEM83 & & \\
\hline 40 & 798 & 15 & rs11854401 & $93,929,730$ & rs8042369 & $93,957,898$ & 8 & UNQ9370//LOC728292 & & \\
\hline 41 & 806 & 16 & rs17606532 & $72,333,127$ & rs12924285 & $72,653,326$ & 13 & LOC390739, LOC645478,UNQ9370 & $16 \mathrm{q} 22.2$ & * \\
\hline 42 & 819 & 17 & chr17:4396 & $43,965,129$ & rs71375338 & $44,332,793$ & 5 & MAPT & $17 q 21.31$ & \\
\hline 43 & 824 & 18 & rs4144756 & $39,305,154$ & rs4144756 & $39,305,154$ & 1 & KC6, PIKЗC3 & & \\
\hline 44 & 825 & 19 & rs13886705 & $37,439,641$ & rs13886705 & $37,439,641$ & 1 & ZNF569 & & \\
\hline 45 & 826 & 20 & rs6047225 & $21,054,496$ & rs6035892 & $21,549,424$ & 280 & $\begin{array}{l}\text { KIZ,RPS15AP1,MRPS11P1,RPL24P2,NKX2-2, } \\
\text { PAX1,GSTM3P,XRN2,NKX2 }\end{array}$ & $20 p 11.23$ & $*$ \\
\hline 46 & 1106 & 20 & rs11480060 & $54,230,218$ & rs11480060 & $54,230,218$ & 1 & RPL12P4,CBLN4 & & \\
\hline 47 & 1107 & 21 & rs11775706 & $36,927,870$ & rs14491176 & $37,255,329$ & 2 & RUNX1 & & \\
\hline
\end{tabular}

*, Regions where SNPs were associated with either ADHD or ASD; BP1 and BP2 are position for flanking SNP1 and SNP2.

Of the total 1,108 SNPs shared by ADHD and ASD, 1,095

SNPs were mapped to 63 genes including intergenic reg- ions (Table S6) and 912 SNPs (82.3\%) were annotated based on the dbSNP[19]. We noted that five SNPs were 
non-synonymous (Table S7); 33 SNPs (3.62\%) are located at transcriptional factor binding site (TFBS), mostly at 20q11.23-11.22 involving three loci at RPL24P//C20orf19, C20orf9, and RPS15AP1//XRN2(Table S8); 55 SNPs $(6.03 \%)$ are conserved in vertebrates and mostly located at 5 genomic regions with CNVs (Table S9). These predicted functional SNPs were located at MAST2, MANBA, SERPINA, C20orf9, FOXP1, TMEM161B-AS1, GBF1/|NFKB2, $P T B P 2, P K P 4, N U D T 12 / / R A B 9 P 1, B B X$, and EDNRB//POU$F 4$. Other SNPs shared by ADHD and ASD were located at loci, including JMJD2A, PTPRF, ST3GAL3, SNX7, SGOL1 |/VENTXP7, EEFSEC||DNAJB8, MAGI1, RSRC1, TNIP2// SH3BP2, KCTD16/|PRELID2, COL19A1, FOXP2, C8orf68, GFRA2, RSU1, FAM76B, PRKD1, NPAS3, TMEM83, MAPT, and RPL12P4//CBLN4 (Table 3). All these 63 genes together annotated a few biological processes, cellular component, and molecular functions, but none of these functional annotations and clustering were significant after multiple testing correction (Table S10).

\section{Genetic variants shared by all five disorders}

Through a combined analysis of 6,391,075 SNPs available common to all five studies, we identified 713 SNPs at 47 genes in 24 genomic regions (defined as above) shared by all five disorders at genome-wide significance (Figure 2A). We note that no SNPs were associayed with schizophrenia (Figure 2B), Bipolar (Figure 2D) or ASD (Figure 2E); but SNPs NUDT12//RAB9P on 5q21.2-21.3 and 10q24-25 have been associated with ADHD (Figure 2C) and MDD (Figure 2E), respectively. SNPs at NUDT12 //RAB9P appear in strong LD in multiple original studies (Figure 2 C, E, F) and SNPs at 10q24-25 were mapped to multiple genes including BLOC1S2, CHUK, CWF19L1, ERIN1, PKD2L1, and SORCS3. The locus 10q24 has been identified in a previous genome-wide analysis of shared effect by five psychiatric disorders.
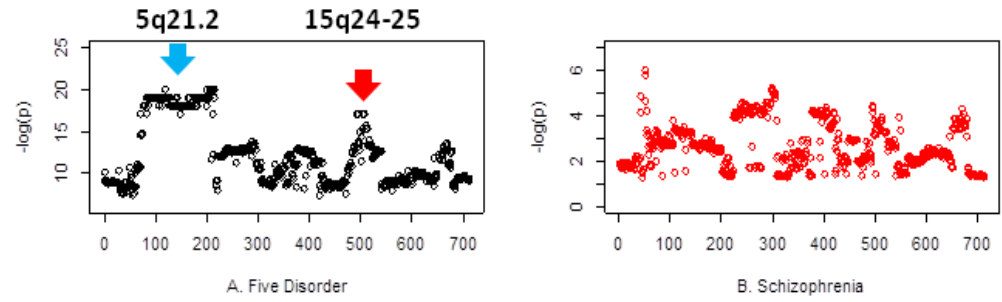

GWAS hits for ADHD, 15q24-25
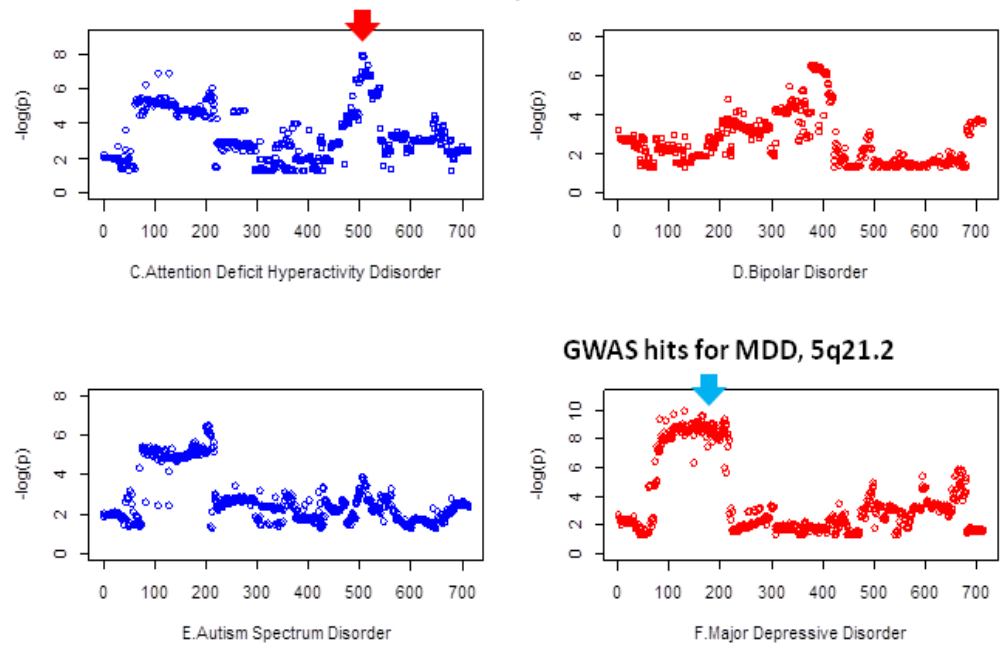

Figure 2. Scatter plots of $p$ values (-log) for 713 SNPs shared by five disorders at genome-wide significance and signals for an individual disorder (X-axis is the SNPs in the chromosomal order ). A) Combined signals for SNPs shared by five disorders; B) Signals with schizophrenia; C) Signals association with ADHD, and SNPs at 15q24-25 associated with ADHD at genome-wide significance; D) Signals for bipolar disorder; E) Signals for ASD; F) Signals for MDD, many SNPs at 5q21.2 were associated with MDD.

In the 22 novel genomic regions that did not contain an SNP associated with the particular disorder at genomewide significance, six SNPs, including rs7945989 at PKP4, rs9375138 at C6orf167,rs12376855 at PCSK5, rs67525828 at CIZ1,rs9804545 at CWF19L2,rs3099047 at CAST-
$P E R 2$, were shared by the five disorders (Table 4). Except for CACNB2 that has been found shared by five disorders, we found additional 40 gene loci in the remaining regions, including A2BP1, RIMS1, DFNA5, CNTN4, MPP6, PLC1, RFTN2, SLC30A9, PAQR3, ERLIN1, SORCS3, ZNF584, 
and ZNF132. The detailed estimates of flanking SNPs at the total 24 regions are in the supplementary (Table S11).

Of the 24 genomic regions that harbored SNPs shared by the five disorders, all had been reported with CNVs associated with ASD (only 15q15.3 known for schizophrenia) and more than half were identified with expression quantitative trait loci (eQTLs) (Table S12). About two-thirds of the CNVs disrupted genes; for example, the CNV at 3p26.3 were associated with CNTN6 and CNTN4, 6q13 with RIMS1, 7p15.3 with MPP6 and DFNA5, 16p13.3 with A2$B P 1$, and 19q13.43 with ZNF584. In addition, we identified SNPs in more than 13 genomic regions as eQTLs in human tissues through cis-association analysis of the flanking SNPs or alternatives with mRNA expression of the mapped genes. The eQTLs affected gene expression of almost 20 genes, including ANKRD44, SF3BL, PLCL1, RFTN2, SLC30A9, PAQR3, NAA11, MPP6, DFNA5, CIZ1, DMM1, C9orf16, BLOC1S2, SORCS3, CWF19L2, STRCP1, ZN584, $R P S S$, and SLC27A5 (Table 4). Additionally, we found that SNP rs3099047 at CATSPER2 had cis-association with about 19 genes in the same region.

Further, we noted that 387 of 713 SNPs shared by five disorders were discovered neither in the analysis of three adult disorders or ADHDASD. However, they were involved in the majority of the genomic regions where the shared 713 SNPs are located (Table S13). We also noted that 146 SNPs were shared by both the three adult-onset disorders and ADHDASD (Figure S1), and they were located at a $400-\mathrm{kb}$ intergenic region NUDT12//RAB9P1 on 5q21.2 and SORCS3 on 10q24-25. The SORCS3 encodes a type-I receptor transmembrane protein, a member of the vacuolar protein sorting 10 (VSP10) receptor family, and had a biased expression in the human brain tissues of the Human Protein Atlas dataset.

\section{DISCUSSIONS}

We conducted a genome-wide association combined analysis of five major psychiatric disorders with an identification of 336 loci shared by three adult psychiatric disorders, 63 loci by ADHD and ASD, and 47 independent loci in 24 genomic regions associated with the combined all five disorders. The more loci shared among three adult disorders were consistent with a recent study that the overlapping gene expression pattern in human brains that SCZ, BD, and MDD have a moderate to high level of transcriptome correlation, but a low correlation with ASD, indicated[23]. Our study reveal a different genetic architecture, in particular the XMHC region, but some shared common variants between the childhood and adultonset psychitric disorders.

In the present study, we found a substantial heterogeneity of genetic variants shared by three adult-onset psychiatric disorders and two childhood disorders. The analysis of a large number of SNPs across the genome indicated that the xMHC SNPs shared by three adulthood disorders had stronger associations with SCZ and MDD than bipolar disorder, but the two childhood disorders did not share them. In addition, three adulthood psychiatric disor-ders shared a number of non-xMHC loci including $D R$ -
D2, GRIN2A, GABRA1, GABRG2, KCNB1, and CACNAI1, in particular CACNA1C, TSNARE1, KLC1, MYTIL that have been found differentially methylated in the human prefrontal cortex between schizophrenia and controls[24], suggesting that these variants may be associated with the risk of the adulthood psychiatric disorders through an epigenetic mechanism. However, none of these genes were found overlapped with that mapped by the SNPs shared by the two childhood disorders. Further, among SNPs at 63 loci shared by ADHD and ASD, only two loci at NUDT12//RAB9P1 and SORCS3 were common to the three adult psychiatric disorders. Given that, $80 \%$ of SNPs shared by two childhood disorders are located at genomic regions with CNVs known for ASD; it may support that CNVs contribute to the genetic causes of two childhood disorders significantly, at least for ASD[25]. However, this study was not aimed to focus on CNVs or rare mutations, which have been implicated for the biology of autism spectrum disorder[26].

Despite the noted heterogeneity for the loci between adult-onset and childhood disorders, new novel or specific loci were identified for five disorders, and they indicate that neurodevelopmental genes may play a role in the development of a spectrum of major psychiatric disorders. First, all of the loci shared by the five disorders were located at genomic regions with CNVs reported for ASD. Previous studies have found that both microdeletions and microduplications greater than 100-kilobases disrupt genes in the neurodevelopmental pathway in schizophrenia[27]. Several genes identified are worthy of mention here. $A 2 B P 1$ has been identified as the top candidate gene in one of two network modules revealed through transcriptome analysis of differentially expressed genes between autism and controls in post-mortem human brains [28]. Disruption of CNTN4 has been shown to result in developmental delay and contributing to the $3 p$ deletion syndrome [29]. CATSPER2 encodes a protein in the family of cation channel proteins that localize to the flagellu$m$ of spermatozoa; defects at this locus cause male infertility, and further a single SNP rs3099047 at CATSPER2 was shown as a significant cis-association with nearly 20 genes in the GTEx dataset. Moreover, we noted that 102 SNPs in a 5-Mb region on 10q24.31-25.1 were mapped to multiple genes and they were eQTLs for BLOC1S2 and SO$R C S 3$ in multiple human tissues; and 2-3 Mb de novo deletions within this region have been detected in individuals with mental retardation and multiple congenital anomalies [30]. All these findings support that the genetic variants shared by the five disorders play a role in neurodevelopment and may through this general mechanism contribute to risk for all psychiatric disorders.

Further, the loci shared by five disorders may have implications for common neurodegenerative disorders such as Alzheimer's disease (AD) and Parkinson's disease (PD). Of the loci shared by five psychiatric disorders, genetic variants at SOCRS3 and CACNB2 have been assoc- 
Table 4. Genomic regions and loci annotated by 713 SNPs common to five disorders at genome-wide significance $\left(\mathrm{P}<5 \times 10^{-08}\right)$.

\begin{tabular}{|c|c|c|c|c|c|c|c|c|c|c|c|}
\hline Reg & ID & $\mathrm{Chr}$ & SNP1 & BP1 & SNP2 & BP2 & \#SNP & Annotated genes or loci & $\mathrm{eQTL}^{*}$ & CNVs & \\
\hline 1 & 1 & 1 & rs904307 & 30473551 & rs1874784 & 30507893 & 41 & PTPRU||MATN1 & & $1 \mathrm{p} 35.2$ & \\
\hline 2 & 42 & 1 & rs28374258 & 190949551 & rs12139300 & 191010445 & 2 & FAM5C//RGS18 & & $1 \mathrm{q} 31.2$ & \\
\hline 3 & 44 & 2 & rs79452989 & 159452935 & & 159452935 & 1 & PKP4 & & $2 \mathrm{q} 24.1$ & \\
\hline 4 & 45 & 2 & rs711810 & 176888760 & rs6755092 & 176906255 & 5 & KIAA1715/|EVX2 & AC009336.24 & $2 \mathrm{q} 31.1$ & \\
\hline 5 & 50 & 2 & rs13404366 & 198522632 & rs2033570 & 198952637 & 4 & PLCL1,RFTN2 & ANKRD44, SF3B1, PLCL1, RFTN2 & $2 \mathrm{q} 33.1$ & \\
\hline 6 & 54 & 3 & rs6778940 & 2571220 & rs75345673 & 2576606 & 4 & CNTN4 & & $3 \mathrm{p} 26.3$ & \\
\hline 7 & 58 & 4 & rs1454607 & 33643360 & rs1454608 & 33643627 & 2 & RP11-79E3.2 & & $4 \mathrm{p} 15.1$ & \\
\hline 8 & 60 & 4 & rs6846961 & 42176060 & rs11930133 & 42184374 & 3 & BEND4, SLC30A9 & SLC30A9, BEND4 & $4 \mathrm{p} 13$ & \\
\hline 9 & 63 & 4 & rs 13108290 & 80198876 & rs17441732 & 80221215 & 8 & LINC01088,PAQR3/|ARD1B & LINC01008, PAQR3, NAA11(ARD1B) & $4 q 21.21$ & \\
\hline 10 & 71 & 5 & rs7716818 & 103684787 & rs325528 & 104048590 & 146 & NUDT12||RAB9P1 & RP11-6N13.1 & $5 q 21.2$ & \\
\hline 11 & 217 & 6 & rs16880300 & 50384635 & rs6930867 & 50936376 & 80 & DEFB112, TFAP2D, FTHP1, FPS17L4, TFAP2B & & 6p12.3 & \\
\hline 12 & 297 & 6 & rs9360557 & 73132745 & rs9351918 & 73149101 & 9 & RIMS1/|LOC643067 & & $6 \mathrm{q} 13$ & \\
\hline 13 & 306 & 6 & rs9375138 & 98518518 & & 98518518 & 1 & C6orf167||LOC100129158 & & 6q16.1 & \\
\hline 14 & 307 & 7 & rs2529055 & 24590331 & rs12154649 & 24832129 & 114 & CTRB1,DFNA5, OSBPL3, MPPG, NPY & MPP6, DFNA5 & $7 \mathrm{p} 15.3$ & \\
\hline 15 & 421 & 9 & rs12376855 & 78591275 & & 78591275 & 1 & PCSK5 & & 9q21.13 & \\
\hline 16 & 422 & 9 & rs67525828 & 130953511 & & 130953511 & 1 & CIZ1 & CIZ1, DNM1, C9orf16, LCN2, CERCAM & $9 q 34.11$ & \\
\hline 17 & 423 & 10 & rs58703423 & 18540122 & rs7091833 & 18660333 & 22 & CACNB2 & RP11-499P20.2 & $10 \mathrm{p} 12.33$ & \\
\hline 18 & 445 & 10 & rs12769818 & 1019155629 & rs13854010 & 107329835 & 102 & BLOC1S2,CHUK,CWF19L1, ERLIN1, PKD2L1, SORCS3 & BLOC1S2, SORCS3, PKDZI1 & 10q24.31-q25.1 & ** \\
\hline 19 & 547 & 11 & rs9804545 & 107278817 & & 107278817 & 1 & CWF19L2 & CWF19L2 & $11 \mathrm{q} 22.3$ & \\
\hline 20 & 548 & 15 & rs3099047 & 43926033 & & 43926033 & 1 & CATSPER2 & $\begin{array}{l}\text { AC011330.5, ADALL, CATSPER2, CATSPER2P1, CCNDBP1, } \\
\text { CKMT1A, ELLL, LCMT2, PDIA3, RNU6-554P, SERF2, } \\
\text { STRC,STRCP1,TGM5,TGM7,TP53BP1,WDR76,ZSCAN29 }\end{array}$ & $15 \mathrm{q} 15.3$ & \\
\hline 21 & 549 & 16 & rs61547418 & 6345040 & rs12448420 & 6346613 & 14 & $A 2 B P 1$ & & $16 q 13.3$ & \\
\hline 22 & 563 & 18 & rs4890712 & 38903507 & rs72893943 & 39318793 & 89 & KC6|/NPM1P1,MIR924||KC6 & $R P 11-142 I 20.1$ & $18 \mathrm{q} 12.3$ & \\
\hline 23 & 652 & 18 & rs7505145 & 50711776 & rs8091083 & 50870391 & 28 & $D C C$ & $D C C$ & $18 \mathrm{q} 21.2$ & \\
\hline 24 & 680 & 19 & rs73060258 & 58902954 & rs12981875 & 58935130 & 34 & FLJ39005, ZNF584, ZNF132 & $\begin{array}{l}\text { ZFN584, ZNF132, RPS5, SLC27A5, AC016629.3, } \\
\text { CHMP2A, CTD-2619J13.14, ZNF324, ZNF446, ZNF497 }\end{array}$ & 19q13.43 & \\
\hline
\end{tabular}

\#SNP, the number of SNPs shared by five psychiatric disorders in the genomic region;

*, eQTLs were tested mostly for the flanking SNPs or alternative SNPs in the loci at each region;

**, of this region, 2-3-Mb size of de novo deletions have been detected with mental retardation and multiple congenital anomalies (MCA) in multiple populations;

Highlighted, SNPs associated with the individual disorder at genome-wide significance;

SNP1 and SNP2 are flanking SNPs for individual region; and BP1 and BP2 are corresponding position (hg19). 
iated with Alzheimer's disease, and both genes had a biased expression in normal brain tissues of the Human Protein Atlas RNA-Seq data. Knockdown of SORCS3 in cell culture leads to an increase in amyloid precursor protein (APP) processing, and APP may be as a mediator of the synapse pathology in the AD [31]. Common genetic variants at CACNB2 have been associated with $\mathrm{AD}$ or through a within-locus SNP by SNP interaction [32]. In addition, we identified five SNPs at microtubule-associated protein tau (MAPT) that were shared by ADHD and ASD. Common genetic variants at MAPT have been associated with the risk of the late-onset $\mathrm{AD}$, and also of $\mathrm{PD}$ in an early large family-based study [33], and shared by AD and PD[34]. Mutations in MAPT have also been detected in the early-onset AD and MAPT-related disorders, a group of neurological disorders including frontotemporal dementia with parkinsonism-17 (FTDP-17), progressive supranuclear palsy (PSP), cortico-basal degeneratetion (CBD), and mild late-onset parkinsonism, dementia with epilepsy.

Finally, it is worth highlighting that our study identified multiple shared genes CACNA1C, TSNARE1, KLC1, and $M Y T I L$ for three adult disorders that have been found differentially methylated in the human prefrontal cortex between schizophrenia and controls in a recent study [24]. Findings of genetic loci would provide a clue about what kind of environment would have caused the high methylation at individual genes associated with psychiatric disorders or a biological basis for conducting geneenvironment interaction analyses. While the collection of environmental data is essential for a complete study of the etiology for common human disorders in the future, the effect that environmental factors exert on humans has to be through the human genome. It would be a great challenge to measure environmental factors across lifespan compared to measure the variants across the whole genome. What is more, most of the lifestyle and behavioral factors are not exogenous, and genetic variants might influence them. In addition to the further neurobiological study, our study provides a manageable list of anchors from which to investigate epigenetic mechanism or gene-gene interaction on the development of neuropsychiatric disorders.

In summary, we identify a sizeable number of genetic variants shared by three psychiatric disorders diagnosed during adulthood, by two childhood disorders, and by all five disorders. These variants indicate genetic heterogeneity but also point to the genetic etiology of neurodevelopment in five major psychiatric disorders or other neurological disorders. Our study provides new insights into genetic etiology and may have important implications for precision neuropsychiatry or medicine.

\section{SUPPLEMENTARY MATERIALS}

Published as e-cintent at the journal's website. (https: //www.gcatresearch.com).

Figure S1 and Table S1-S13

\section{CONFILCTS OF INTERESTS}

The authors declare no conflict of interest regarding the publication of this paper.

\section{ACKNOWLEDGEMENTS}

This work was supported by grants from the National Natural Science Foundation of China (No. 81525007 and No.81730036, $\mathrm{KX}$ ), the China Scholarship Council (LX), direct funding of Lieber Institute for Brain Development (DRW), the Haiju Scholarship through Peking University Beijing Huilongguan Hospital and Global Clinical and Tranlaslational Research Institute (FZ). We also thanks the Psychiatric Genetics Consotium, which makes the data available.

\section{REFERENCES}

1. Hardy, J. and A. Singleton, Genomewide association studies and human disease. N Engl J Med, 2009. 360 (17): p. 1759-68.

2. Scott, L.J., et al., A genome-wide association study of type 2 diabetes in Finns detects multiple susceptibility variants. Science, 2007. 316(5829): p. 1341-5.

3. Duerr, R.H., et al., A genome-wide association study identifies IL23R as an inflammatory bowel disease gene. Science, 2006. 314(5804): p. 1461-3.

4. Schizophrenia Working Group of the Psychiatric Genomics, C., Biological insights from 108 schizoph-renia-associated genetic loci. Nature, 2014. 511 (7510): p. 421-7.

5. Manolio, T.A., In Retrospect: A decade of shared genomic associations. Nature, 2017. 546(7658): p. 360361.

6. Hyde, C.L., et al., Identification of 15 genetic loci associated with risk of major depression in individuals of European descent. Nat Genet, 2016. 48(9): p. 1031-6.

7. Wray, N.R., et al., Genome-wide association analyses identify 44 risk variants and refine the genetic architecture of major depression. Nat Genet, 2018. 50(5): p. 668-681.

8. Geschwind, D.H. and J. Flint, Genetics and genomics of psychiatric disease. Science, 2015. 349(6255): p. 1489-94.

9. International Schizophrenia, C., et al., Common polygenic variation contributes to risk of schizophrenia and bipolar disorder. Nature, 2009. 460(7256): p. 748-52.

10. Solovieff, N., et al., Pleiotropy in complex traits: challenges and strategies. Nat Rev Genet, 2013. 14(7): p. 483-95.

11. Cross-Disorder Group of the Psychiatric Genomics, C., Identification of risk loci with shared effects on five major psychiatric disorders: a genome-wide analysis. Lancet, 2013. 381(9875): p. 1371-1379.

12. Lee, S.H., et al., Genetic relationship between five psychiatric disorders estimated from genome-wide SNPs. Nat Genet, 2013. 45(9): p. 984-94.

13. The US National Research Council Committee. A Framework for Developing a New Taxonomy of Disease, in Toward Precision Medicine: Building a Knowledge Network for Biomedical Research and a New Taxonomy of Disease. 2011: Washington (DC).

14. Collins, F.S. and H. Varmus, A new initiative on precision medicine. N Engl J Med, 2015. 372(9): p. 793-5. 
15. Bipolar, D., et al., Genomic dissection of bipolar disorder and schizophrenia, including 28 subphenotypes. Cell, 2018. 173(7): p. 1705-1715 e16.

16. Demontis, D., et al., Discovery of the first genome-wide significant risk loci for ADHD. bioRxiv 2017 (doi: $10.1101 / 145581$ ).

17. Grove, J., et al., Common risk variants identified in autism spectrum disorder. bioRxiv, 2017. doi:10. 1101 /224774.

18. Sullivan, P.F., et al., Genomewide association for schizophrenia in the CATIE study: results of stage 1 . Mol Psychiatry, 2008. 13(6): p. 570-84.

19. Xu, Z. and J.A. Taylor, SNPinfo: integrating GWAS and candidate gene information into functional SNP selection for genetic association studies. Nucleic Acids Res, 2009. 37(Web Server issue): p. W600-5.

20. Huang da, W., B.T. Sherman, and R.A. Lempicki, Systematic and integrative analysis of large gene lists using DAVID bioinformatics resources. Nat Protoc, 2009. 4(1): p. 44-57.

21. de Bakker, P.I., et al., A high-resolution HLA and SNP haplotype map for disease association studies in the extended human MHC. Nat Genet, 2006. 38(10): p. 1166-72.

22. Horton, R., et al., Gene map of the extended human MHC. Nat Rev Genet, 2004. 5(12): p. 889-99.

23. Gandal, M.J., et al., Shared molecular neuropathology across major psychiatric disorders parallels polygennic overlap. Science, 2018. 359(6376): p. 693-697.

24. Pidsley, R., et al., Methylomic profiling of human brain tissue supports a neurodevelopmental origin for schizophrenia. Genome Biol, 2014. 15(10): p. 483.

25. Sanders, S.J., et al., Insights into autism spectrum disorder genomic architecture and biology from 71 risk loci. Neuron, 2015. 87(6): p. 1215-1233.
26. State, M.W. and N. Sestan, Neuroscience. The emerging biology of autism spectrum disorders. Science, 2012. 337(6100): p. 1301-3.

27. Walsh, T., et al., Rare structural variants disrupt multiple genes in neurodevelopmental pathways in schizophrenia. Science, 2008. 320(5875): p. 539-43.

28. Voineagu, I., et al., Transcriptomic analysis of autistic brain reveals convergent molecular pathology. Nature, 2011. 474(7351): p. 380-4.

29. Fernandez, T., et al., Disruption of contactin 4 (CNTN4) results in developmental delay and other features of $3 p$ deletion syndrome. Am J Hum Genet, 2004. 74(6): p. 1286-93.

30. Hayashi, S., et al., Clinical application of array-based comparative genomic hybridization by two-stage screening for 536 patients with mental retardation and multiple congenital anomalies. J Hum Genet, 2011. 56 (2): p. 110-24.

31. Schreurs, A., A. Latif-Hernandez, and A. Uwineza, Commentary: APP as a Mediator of the Synapse Pathology in Alzheimer's Disease. Front Cell Neurosci, 2018. 12: p. 150.

32. Liang, X., et al., Genomic convergence to identify candidate genes for Alzheimer disease on chromosome 10. Hum Mutat, 2009. 30(3): p. 463-71.

33. Martin, E.R., et al., Association of single-nucleotide polymorphisms of the tau gene with late-onset Parkinson disease. JAMA, 2001. 286(18): p. 2245-50.

34. Desikan, R.S., et al., Genetic overlap between Alzheimer's disease and Parkinson's disease at the MAPT locus. Mol Psychiatry, 2015. 20(12): p. 1588-95.

How to cite this article:

Xia, L, Xia, K, Weinberger, DR, Zhang F. Common genetic variants shared among major psychiatric disorders: A genome-wide combined analysis. Glob Clin Transl Res. 2019; 1(1):21-30

Copyright (C) 2019 by the Global Clinical and Translational Research. 